\title{
Reception and Operational Processing of Satellite Data in Regional Centre for Remote Sensing at FRC KSC SB RAS (Krasnoyarsk, Russia)
}

\author{
Oleg E. Yakubailik', Victor Yu. Romas'ko ${ }^{2}$, and Evgeny A. Pavlichenko ${ }^{2}$ \\ ${ }^{1}$ Federal Research Center "Krasnoyarsk Science Center of the Siberian Branch of the Russian Academy \\ of Sciences", Institute of Computational Modeling SB RAS, Krasnoyarsk, Russia \\ oleg@icm.krasn.ru \\ ${ }^{2}$ Federal Research Center "Krasnoyarsk Science Center of the Siberian Branch \\ of the Russian Academy of Sciences", Krasnoyarsk, Russia
}

\begin{abstract}
The article discusses the main problems and trends in the development of modern systems of reception, storage and operational processing of satellite data. There is a sharp increase in the capabilities of satellite systems, a significant increase in the volume of satellite information and its availability, the development of data processing and presentation technologies, the use of web technologies. The data sources of modern earth remote sensing systems and the peculiarities of their practical use are analyzed. It is shown that satellite stations receiving data in the X-band at a frequency of $8 \mathrm{GHz}$ are an effective way to obtain operational information from meteorological satellites. The architecture of the hardware and software of the satellite receiving complex of the FRC KSC SB RAS is considered, the spacecraft and their sensors from which the data are received are listed. The results of the work performed on the creation of specialized software for information and analytical systems of operational satellite monitoring are presented.
\end{abstract}

Keywords: satellite monitoring, geoportal, spatial data processing, monitoring system, UniScan, remote sensing, web service, catalog of satellite images 


\title{
ПРИЕМ И ОПЕРАТИВНАЯ ОБРАБОТКА СПУТНИКОВЫХ ДАННЫХ В РЕГИОНАЛЬНОМ ЦЕНТРЕ ДЗЗ ФИЦ КНЦ СО РАН
}

\author{
О.Э. Якубайлик ${ }^{1}$, В.Ю. Ромасько, Е.А. Павличенко ${ }^{2}$ \\ ${ }^{1}$ Федеральный исследовательский центр «Красноярский научный центр СО РАН», \\ Институт вычислительного моделирования СО РАН, Красноярск, Россия \\ oleg@icm.krasn.ru
}

${ }^{2}$ Федеральный исследовательский центр «Красноярский научный центр СО РАН», Красноярск, Россия

В статье обсуждаются основные проблемы и тенденции в развитии современных систем приема, хранения и оперативной обработки спутниковых данных. Отмечается резкий рост возможностей спутниковых систем, значительное увеличение объемов спутниковой информации и ее доступности, развитие технологий обработки и представления данных, использование веб-технологий. Анализируются источники данных современных систем дистанционного зондирования Земли, особенности их практического использования. Показано, что спутниковые станции, осуществляющие прием данных в Xдиапазоне, на частоте 8 ГГц, являются эффективным способом получения оперативной информации с метеорологических спутников. Рассматривается архитектура аппаратно-программного обеспечения спутникового приемного комплекса ФИЦ КНЦ СО РАН, перечислены космические аппараты и их сенсоры, с которых ведется прием данных. Представлены результаты выполненных работ по созданию специализированного программного обеспечения для информационно-аналитических систем оперативного спутникового мониторинга.

Ключевые слова: спутниковый мониторинг, геопортал, обработка пространственных данных, система мониторинга, УниСкан, ДЗ3, веб-сервис, каталог спутниковых снимков

\section{Введение}

Географические особенности Красноярского края (протяженная территория с разнообразным рельефом и климатом, высокая доля слабо освоенных и труднодоступных зон, большие запасы природных ресурсов и другие факторы) объективно приводят к необходимости использования систем дистанционного зондирования Земли (ДЗ3) и различных тематических информационных продуктов на их основе. Интеграция получаемых данных ДЗ3 с реальными процессами обеспечения жизнедеятельности органов государственной власти и населения приобретает значение стратегического фактора для дальнейшего ускорения социальноэкономического развития Красноярского края.

Одним из этапов решения актуальных задач по обеспечению эффективного использования результатов космической деятельности в Красноярском крае стало приобретение и запуск в эксплуатацию в 2017 г. нового спутникового приемного комплекса ФИЦ КНЦ СО РАН на базе станции УниСкан-36 компании СКАНЭКС, который обеспечивает возможность приема данных Д33 со всех актуальных низкоорбитальных метеорологических спутников среднего пространственного разрешения (американские TERRA, AQUA, Suomi NPP, NOAA-20, китайские FengYun-3).

Можно считать, что общая схема организации процессов обработки данных ДЗЗ уже достаточно устоялась; основные блоки включают прием и архивацию, первичную и 
тематическую обработку $[1,2]$. Если на первых этапах развития систем мониторинга при построении конкретной системы в ней приходилось реализовывать все этапы обработки данных Д33 и полностью создавать для этого специальную инфраструктуру, то в настоящее время более эффективным и целесообразным с разных точек зрения считается использования специализированных центров хранения, обработки и анализа данных $[3,4]$. В исследованиях и разработках, проводимых в настоящее время, рассматриваются различные аспекты реализации соответствующего программного обеспечения [5-7].

Стоит отметить, что в области, связанной с использованием спутниковых данных дистанционного зондирования Земли (ДЗ3) при решении различных научных и прикладных задач, в настоящее время происходят существенные изменения:

- Резко возросли возможностях спутниковых систем Д3З; за счет увеличения числа космических аппаратов Д3З стало возможно организовывать мониторинг быстро протекающих процессов. За последние 5 лет число спутников увеличилось примерно на порядок, возросла доступность данных высокого разрешения (в частности, появились зарубежные снимки с разрешением до 3 м/пиксел, которые можно получать бесплатно для научных исследований, хотя и с некоторыми ограничениями; для ряда категорий потребителей спутниковой информации организован бесплатный доступ к данным с российских спутников с разрешением до 1 м/пиксел).

- Возросло число спутниковых систем Д33, обладающих «измерительными» свойствами, т.е. обеспечивающих получение не только качественной, но и хорошо калиброванной количественной информации о различных объектах, процессах и явлениях.

- Повысился уровень доступности информации. Данные многих спутниковых систем стали свободно распространяться - как через сеть Интернет, так и непосредственно со спутников, в незашифрованном виде.

- Значительный рост объемов спутниковой информации и развитие требований и стоимости систем их приема и первичной обработки привели к усилению тенденции перехода от использования персональных систем обработки ДЗ3 к сервис-ориентированным решениям на основе региональных специализированных центров ДЗ3.

Наряду с экстенсивным развитием спутниковых систем ДЗ3 в последнее десятилетие наблюдается и достаточно резкий рост объемов спутниковых данных, получаемых с отдельных космических аппаратов, а также номенклатуры и суммарных объемов информационных продуктов, формируемых на основе обработки этих данных, т.е. скорость роста объемов информации превышает скорость роста числа систем ДЗ3. Так, например, объемы архивов данных Д33 в NASA и NOAA, накапливаемые за год, увеличились более чем на порядок: от почти 1 Петабайта в 2000 г. до более 10 Петабайт в 2011 г., хотя число спутников ДЗ3 увеличилось за это время не более чем в 6 раз. Ввод новых данных ДЗ3 в систему архивации в 2018 г. по планам NOAA составит 20 петабайт/год, а общий их объем - более 100 Петабайт. При этом объем данных из расчета на один спутник вырастет в 100 раз. Аналогичные планы по наращиванию группировки ДЗ3 и быстрому росту объемов информации ДЗЗ приняты к реализации и в России (Федеральная космическая программа России на 2016 - 2025 годы).

Отмечается резкий рост возможностей спутниковых систем, значительное увеличение объемов спутниковой информации и ее доступности, развитие технологий обработки и представления данных, использование веб-технологий.

\section{Источники данных современных систем спутникового мониторинга}

Оборудование современных систем ДЗ3 традиционно строится вокруг главных источников данных ДЗ3. Получать данные ДЗ3 можно 1) по заказу у официальных поставщиков данных Д33 (как правило, это данные высокого пространственного разрешения), 2) по Интернету из бесплатных источников и, наконец, 3) непосредственным приемом данных со спутников, работающих в режиме вещания. Бесплатная информация низкого и среднего разрешения, а иногда, в последнее время, и высокого, большей частью доступна для скачивания с официальных ресурсов, например, таких как сервис USGS Earth Explorer Геологической службы США [8]. 
Этот способ получения данных в нашей стране постепенно принимает все большее значение, по мере роста скорости и удешевления доступа в Интернет. Однако через подобные сервисы не все спутниковые данные доступны, особенно это касается новых и развивающихся систем. Другой проблемой являются задержки в обработке данных - они не всегда обрабатываются достаточно оперативно - этот фактор может стать принципиальным для задач типа оперативного мониторинга паводков или пожаров. Неприятной, теоретически возможной перспективой таких сервисов является введение платы за использование, пусть и небольшой, но которая полностью может поменять принципы финансового обеспечения работ по обработке данных ДЗЗ.

Проведенный анализ показал, что самым оперативным и надежным вариантом получения данных среднего и низкого разрешения, является собственная (или доступная партнерская) станция приёма космической информации. Из существующих стандартов передачи данных в диапазонах 137 МГц, 1,7 и 8 ГГц последний позволяет передавать максимальный объем информации за сеанс. В зависимости от диаметра антенны, числа частот приема (приемников) и типа фазовой модуляции такая станция способна принимать до 320 Мбит/с [9]. С учётом высоты орбиты полярно-орбитальных спутников порядка 800 км и полосы захвата 2-3 тыс. км зона обзора такой станции представляет из себя приблизительно эллипс размером 5 на 8 тыс. км, вытянутый по долготе. Для станции, расположенной вблизи географического центра России - в Красноярске, обеспечивается обзор приблизительно 80\% территории России дважды в сутки с каждого принимаемого аппарата. Именно такая станция Х-диапазона частот (8 ГГц) и составляет основу спутникового приемного комплекса ФИЦ КНЦ СО РАН.

\section{Обработка спутниковой информации}

Схема аппаратно-программного обеспечения обработки данных ДЗ3 в случае наличия собственной станции приема спутниковых данных состоит как правило из 3 логических групп серверов - приемных, предварительной обработки и тематической обработки, а также автоматизированных рабочих мест для интерактивной тематической обработки (Рис. 1). Системы хранения данных Д33, характеризуемых большими суточными и сеансовыми объёмами, может быть интегрировано в сервера этих логических групп или располагаться отдельно, может быть, как часть большей инфраструктуры. Разделение серверов и процессов на эти три группы логически вытекает из особенностей каждой группы процессов приема и обработки.

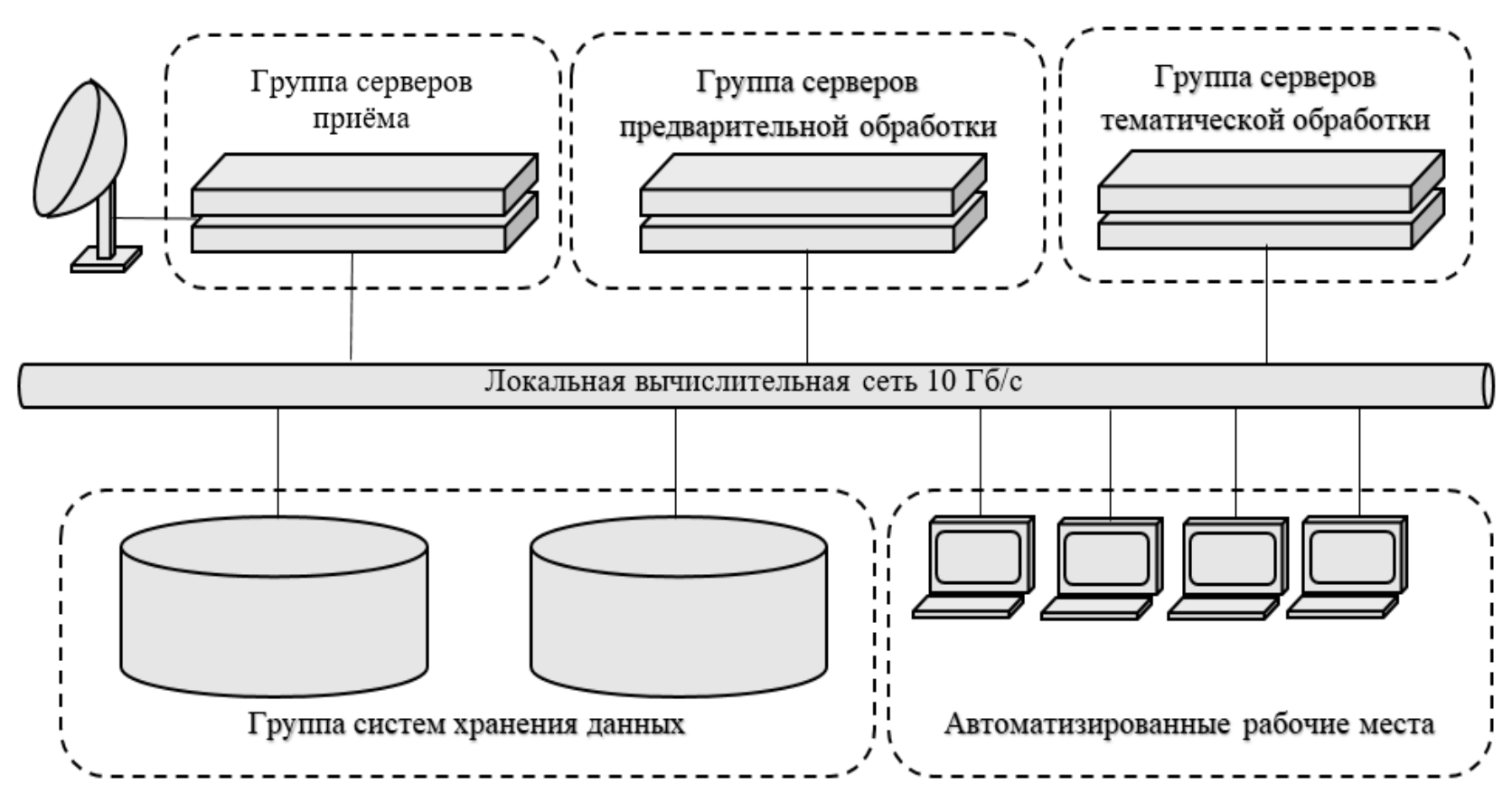

Рис. 1. Схема аппаратно-программного обеспечения спутникового приемного комплекса ФИЦ КНЦ СО РАН 
Структура и основные характеристики приведенных групп серверов:

- Группа серверов приема данных выделена по причине работы в режиме реального времени в моменты приема данных по радиоканалу и поэтому не нагружается никакими дополнительными нагрузками по обработке. Задача серверов этой группы: принять и записать без потерь на дисковую память весь поток данных со всех сеансов. Используемое в ФИЦ КНЦ СО РАН программное обеспечение для приема данных разработано компанией СКАНЭКС поставщиком спутниковой приемной станции. Оно обеспечивает полную автоматизацию всех процессов: скачивание орбитальных элементов, прием сигналов точного времени от GPS, составление расписания на основе заданных приоритетов приема данных с различных космических аппаратов, управление несколькими приемными серверами, и проч. При необходимости оператору предоставляется вся текущая информация о процессе работы станции в реальном времени, в том числе, и картосхема пролёта космического аппарата, и реальный видеопоток с него (если возможно декодирование его на лету).

- Группа серверов предварительной обработки включает процессы географической привязки и радиометрической калибровки принятых данных. Она логически обособлена, т.к. процессы предварительной обработки обязательны для всех космических систем ДЗЗ и выполняются в первую очередь, а также в связи с тем, что они реализуются на программном обеспечении, специфичном для каждой системы Д33, которое предоставляется разработчиками этой системы. Программное обеспечение предварительной обработки, установленное в ФИЦ КНЦ CO PAH, включает в себя три стандартных пакета для данных разных спутниковых систем: TERRA/AQUA - для радиометра MODIS, Suomi NPP/NOAA-20 - для радиометров VIIRS, CrIS, ATMS, Fengyun-3A/B/C - для радиометра MERSI. Все пакеты работают в среде Linux/CentOS на одном сервере. Скрипты обработки запускаются через задаваемый интервал времени (15 минут).

- Третья группа серверов тематической обработки, собственно, и производит целевую обработку данных с получением конечной полезной информации для потребителей. В эту группу попадает, в том числе, тематическое программное обеспечение собственной разработки ФИЦ КНЦ СО РАН. Процессы обработки здесь более однородны и согласованы, а также наиболее часто меняются в следствие разработки новых версий или приложений. В состав этой группы в ФИЦ КНЦ СО РАН включен в том числе веб-интерфейс, построенный на основе геопортальных технологий, как главная точка доступа потребителей к информации Д33 [10-12]. Тематическая обработка данных выполняется параллельно на двух серверах. Для каждого принятого спутникового снимка с метеорологических спутников TERRA/AQUA, Suomi NPP/NOAA-20 генерируется набор мультимасштабных многоканальных обзорных изображений/«квик-луков» (избранные комбинации спектральных каналов, температура поверхности, NDVI) для практически мгновенного отображения информации на веб-странице.

Спектр задач, решаемых на основе получаемых данных дистанционного зондирования Земли, постоянно расширяется. Исследования в области создания систем оперативного спутникового мониторинга, выполняемые в ФИЦ КНЦ СО РАН, предполагают создание новых и, на первом этапе, использование существующих публично доступных программ обработки ДЗ3 [13], в том числе алгоритмов для радиометров MODIS и VIIRS: поиска активных пожаров, оценки содержания аэрозолей, оценки площади гарей, маскирования облачности, определения оптических свойств облачности (температура, высота, фракция и агрегатное состояние верхней границы), атмосферной коррекции видимых и ближних ИК каналов, восстановления вертикальный профилей атмосферы, вычисления NDVI и EVI.

\section{Выводы}

Наблюдаемый в настоящее время значительный рост объема спутниковых данных приводит к необходимости развития методов и технологий построения систем приема, хранения и обработки спутниковой информации. Анализ существующих решений и собственный опыт 
исследований и разработок показывает, что одним из наиболее перспективных подходов в создании систем указанного типа является реализация комплекса взаимосвязанных автономных функционально-ограниченных компонент, взаимодействующих между собой в сервисориентированной архитектуре, с интерфейсом пользователя, построенном на основе технологий геоинформационных веб-систем.

\section{References}

[1] Kashnitskii, A.V., Lupyan, E.A., Balashov, I.V., Konstantinova, A.M. Technology for designing tools for the process and analysis of data from very large scale distributed satellite archives, Atmospheric and Oceanic Optics, 2017, 30, № 1, pp. 84-88. DOI: 10.1134/S1024856017010080

[2] Casu, F., Manunta, M., Agram, P.S., Crippen, R.E. Big Remotely Sensed Data: tools, applications and experiences, Remote Sensing of Environment, 2017, 202, pp. 1-2. DOI: 10.1016/j.rse.2017.09.013

[3] Craglia, M., de Bie, K., Jackson, D., Pesaresi, M., et al. Digital Earth 2020: Towards the vision for the next decade, Int. Journal of Digital Earth, 2012, 5, № 1, pp. 4-21. DOI: 10.1080/17538947.2011.638500

[4] Xu, W., Shao, J., Yu, W., Feng, P. Land observing satellite data center: Big data challenges and a potential solution, Geomatics and Information Science of Wuhan University, 2017, 42, № 1, pp. 7-13. DOI: 10.13203/j.whugis20150175

[5] Adam Lewis, Simon Oliver, Leo Lymburner, Ben Evans, Lan-Wei Wang. The Australian Geoscience Data Cube - Foundations and lessons learned, Remote Sensing of Environment, 2017, 202, pp. 276-292. DOI: 10.1016/j.rse.2017.03.015

[6] A. Ahamed, J. D. Bolten. A MODIS-based automated flood monitoring system for SouthEast Asia, International Journal of Applied Earth Observation and Geoinformation, 2017, 61, pp. 104-117. DOI: $10.1016 /$ j.jag.2017.05.006

[7] Yi Liu, Jiameng Hu, Isaiah Snell-Feikema, Michael S. VanBemmel, Michael C. Wimberly. Software to facilitate remote sensing data access for disease early warning systems, Environmental Modelling \& Software, 2015, 74, pp. 247-257. DOI: 10.1016/j.envsoft.2015.07.006

[8] USGS EarthExplorer. URL: https://earthexplorer.usgs.gov/

[9] Scanex UniScan Receiving station. URL: http://www.scanex.ru/station/uniskan/

[10] Yakubailik O.E., A. Kadochnikov A.A., Tokarev A.V. Applied software tools and services for rapid web GIS development, International Multidisciplinary Scientific GeoConference SGEM2015 Conference Proceedings, 2015, Book 2, 1, pp. 487-494

[11] Yakubailik O.E. The formation of regional spatial data infrastructure, Journal of Siberian Federal University. Engineering \& Technologies, 2016, 9, 7, pp. 979-986. DOI: 10.17516/1999-494X-2016-9-7-979-986.

[12] Yakubailik O.E. Technologies of formation of interactive thematic maps on geoportal, Vestnik komp'iuternykh i informatsionnykh tekhnologii, 2017, 4, pp. 23-28. DOI: 10.14489/vkit.2017.04.pp.023-028 (in Russian)

[13] Coronado P., Brentzel K. NASA Direct Readout for Its Polar Orbiting Satellites. In Earth Science Satellite Remote Sensing Vol. 2: Data, Computational Processing, and Tools, edited by J. Qu, W. Gao, M. Kafatos, R. Murphy, and V. Salomonson, 2017, Beijing: Tsinghua University Press. DOI: 10.1007/978-3-540-37294-3_4 